\title{
Revision of a Derivative-Free Quasi-Newton Method
}

\author{
By John Greenstadt
}

\begin{abstract}
A derivative-free Quasi-Newton (DFQN) method previously published [ $\mathrm{J}$. Greenstadt, Math. Comp., v. 26, 1972, pp. 145-166] has been revised and simplified. The main modification has the effect of keeping all the successive approximants to the Hessian matrix positive-definite. This, coupled with some improvements in the line search, has enhanced the performance of the method considerably. The results of numerical trials on many of the "standard" test functions are displayed, in addition to comparisons with two other methods. These indicate that the present DFQN method is not too far behind that of Gill, Murray and Pitfield, the most efficient one presently known.
\end{abstract}

1. Introduction. The work to be described here is an extension of a previous attempt [1] to devise a derivative-free Quasi-Newton (DFQN) method, which does not make explicit use of difference approximations. Considerable improvements have been made, which have rendered the method much more robust and efficient than before.

As is usual, our problem is to minimize a function $f$ of the argument $x$ (which is a vector with $N$ components). We assume that we have available only the value of $f($ for any $x$ ), but none of its derivatives. Part of our task is to estimate the gradient of $f\left(\equiv\left\{\partial f / \partial x_{i}\right\}\right)$ and its Hessian $\left(\equiv\left\{\partial^{2} f / \partial x_{i} \partial x_{j}\right\}\right)$ using the available function values only. We shall denote the true values of the gradient and Hessian by $\bar{g}$ and $\bar{G}$, respectively, and the estimates by $g$ and $G$. Naturally, our reason for making these estimates is so that we may calculate a good step $\delta$, according to Newton's famous formula:

$$
\delta=-\bar{G}^{-1} \bar{g} \text {. }
$$

When $\bar{G}$ is positive-definite, formula (1.1) will always provide a descent direction, i.e., one in which $f(x)$ initially decreases. The principal difficulty in [1] was that the computed estimate, $G$, was often not positive-definite (even when the true Hessian $\bar{G}$ was). One of the main improvements of the present revision is a reliable way of preventing this mishap.

2. Cycles of Steps. The overall sequence of steps, by which the minimum of $f(x)$ is sought, is partitioned into subsequences, or cycles, of $N$ steps each. ${ }^{1}$ Each such cycle is handled independently of all the others, so that the notation we shall use will, for convenience, ignore the fact that there is really a sequence of cycles.

In fact, we shall refer the various points $\left\{x_{i}\right\}$, reached in a given cycle, to the starting point $\left(x_{0}\right)$ of that cycle. The relative position vector $\tau_{i}$ within this cycle is

Received June 12, 1975 ; revised March 24, 1977.

AMS (MOS) subject classifications (1970). Primary 90C30, 90-04.

Key words and phrases. Unconstrained minimization, Quasi-Newton methods, minimization without derivatives.

1 These were called "major steps" in [1].

Copyright @ 1978, American Mathematical Society 
then defined as follows:

$$
\tau_{i} \equiv x_{i}-x_{0} \quad(i=1, \ldots, N) .
$$

Obviously, $\tau_{0}=0$. Further, we shall mostly regard $f$ as a function of $\tau$, rather than $x$. We shall denote the step vectors within a typical cycle by $\left\{\sigma_{i}\right\}$, with $i=1$, $\ldots, N$. Each step can also be defined in terms of a suitably normalized direction vector $s_{i}$, and a step length $h_{i}$. The sequence of successive relative positions $\left\{\tau_{i}\right\}$ within the cycle is, by definition, given by:

$$
\tau_{i}=\tau_{i-1}+\sigma_{i}
$$

In turn, $\sigma_{i}$ is given by:

$$
\sigma_{i}=h_{i} s_{i}
$$

The step length $h_{i}$ is to be found by a line search along $s_{i}$, starting from $\tau_{i-1}$. For convenience, we parametrize the line through $\tau_{i-1}$, and in the direction $s_{i}$, using the parameter $\alpha_{i}$, so that any position $\tau\left(\alpha_{i}\right)$ along this line is given by:

$$
\tau\left(\alpha_{i}\right) \equiv \tau_{i-1}+\alpha_{i} s_{i}
$$

On this basis, the function $f\left(\tau\left(\alpha_{i}\right)\right)$ can be denoted by $F_{i}\left(\alpha_{i}\right)$, so that

$$
F_{i}\left(\alpha_{i}\right) \equiv f\left(\tau_{i-1}+\alpha_{i} s_{i}\right) .
$$

During any line search, we evaluate $F_{i}\left(\alpha_{i}\right)$ for various values of $\alpha_{i}$, and finally end up with a set of three such values $\left\{\alpha_{i}^{(1)}, \alpha_{i}^{(2)}, \alpha_{i}^{(3)}\right\}$ with the properties:

$$
\begin{gathered}
\alpha_{i}^{(1)}<\alpha_{i}^{(2)}<\alpha_{i}^{(3)}, \\
F_{i}\left(\alpha_{i}^{(1)}\right)>F_{i}\left(\alpha_{i}^{(2)}\right)<F_{i}\left(\alpha_{i}^{(3)}\right) .
\end{gathered}
$$

(It is not necessary, however, that $F_{i}\left(\alpha_{i}^{(2)}\right)$ be the minimum of $F_{i}\left(\alpha_{i}\right)$.)

We define the step length as follows:

$$
h_{i} \equiv \alpha_{i}^{(2)} .
$$

The sequence of directions $\left\{s_{i}\right\}$ is chosen as follows:

(a) At $\tau_{0}$ (the start of the cycle), we assume that we have estimates $g_{0}$ and $G$, to the true values $\bar{g}\left(\tau_{0}\right)$ and $\bar{G}$. The (unnormalized) direction $\delta_{1}$ is calculated by the Newton formula:

$$
\delta_{1}=-G^{-1} g_{0}
$$

(b) The normalized vector $s_{1}$ is calculated by:

$$
s_{1} \equiv \frac{\delta_{1}}{\sqrt{\delta_{1}^{T} G \delta_{1}}}
$$

(where the superscript $T$ indicates the transpose) which results in: ${ }^{2}$

${ }^{2}$ This normalization is feasible because $G$ can be kept positive definite. In [1], a different normalization was necessary. (Note, too, that all vectors are regarded as column matrices.) 


$$
s_{1}^{T} G s_{i}=1 .
$$

(c) The subsequent $s_{i}$ (for $i=2, \ldots, N$ ) are selected recursively, in such a way that they all form a conjugate set with respect to $G$. Thus,

$$
s_{i}^{T} G s_{j}=\delta_{i j} \quad(i, j=1, \ldots, N) .
$$

(In the program used for testing, successive coordinate directions were selected, and the Gram-Schmidt orthogonalization procedure was applied, with $G$ as the weight matrix. The linear independence of each new direction vector was checked.)

With a set of $\left\{s_{i}\right\}$ that satisfy Eq. (2.11), the following considerations prove to be useful: Since the $\left\{s_{i}\right\}$ have been constructed so as to be linearly independent, we can form the nonsingular matrix $S$, whose columns consist of the vectors $\left\{s_{i}\right\}$ as follows:

$$
S \equiv\left\{s_{1}, s_{2}, \ldots, s_{N}\right\} .
$$

Also, we can form the matrix $R$, whose columns consist of the products $\left\{G s_{i}\right\}$ as follows:

$$
R \equiv\left\{G s_{1}, G s_{2}, \ldots, G s_{N}\right\}=G S .
$$

Forming the product $R^{T} S$, we have

$$
R^{T} S=\left\{s_{i}^{T} G s_{j}\right\}=\left\{\delta_{i j}\right\}=I
$$

as a consequence of (2.11). Hence, it is clear that

$$
R^{T}=S^{-1}
$$

and it follows that:

$$
\sum_{i=1}^{N} s_{i} s_{i}^{T} G=S R^{T}=S S^{-1}=I .
$$

3. The Quasi-Newton (QN) Conditions. All QN conditions may be regarded as identities on quadratic functions. Following this viewpoint, we approximate $f(\tau)$ locally $^{3}$ by a quadratic function $Q(\tau)$, defined by

$$
Q(\tau) \equiv Q_{0}+\tau^{T} g_{0}+1 / 2 \tau^{T} G \tau,
$$

where $g_{0}$ and $G$ are the approximations associated with the current cycle. After this cycle has been completed, the information gathered in regard to $f(\tau)$ is to be used to update $g_{0}$ and $G$. (These updates we shall denote by $g_{0}^{*}$ and $G^{*}$.) This will be done in such a way that $Q(\tau)$ will match $f(\tau)$ on every step in the cycle. This updated $Q$ (to be denoted by $Q^{*}$ ) is defined quite analogously to (3.1):

$$
Q^{*}(\tau) \equiv Q_{0}^{*}+\tau^{T} g_{0}^{*}+1 / 2 \tau^{T} G^{*} \tau
$$
cycle.

3“Locally" means: On the set of points $\left\{\tau_{i}\right\}$, (with $i=0, \ldots, N$ ) which make up a 
Along the line defined by (2.4), $Q^{*}(\tau)$ depends only on $\alpha_{i}$, so that for convenience, we shall define a function $R_{i}\left(\alpha_{i}\right)$ as follows:

$$
R_{i}\left(\alpha_{i}\right) \equiv Q^{*}\left(\tau_{i-1}+\alpha_{i} s_{i}\right)
$$

and, by expanding $Q^{*}$, we obtain:

$$
\begin{aligned}
R_{i}\left(\alpha_{i}\right)= & \left(Q_{0}+\tau_{i-1}^{T} g_{0}^{*}+1 / 2 \tau_{i-1}^{T} G^{*} \tau_{i-1}\right) \\
& +\left(s_{i}^{T} g_{0}^{*}+s_{i}^{T} G^{*} \tau_{i-1}\right) \alpha_{i}+1 / 2\left(s_{i}^{T} G^{*} s_{i}\right) \alpha_{i}^{2} .
\end{aligned}
$$

The three expressions in parentheses will be denoted by $a_{i}, b_{i}$ and $c_{i}$, respectively, so that $R_{i}\left(\alpha_{i}\right)$ can be abbreviated to:

$$
R_{i}\left(\alpha_{i}\right)=a_{i}+b_{i} \alpha_{i}+1 / 2 c_{i} \alpha_{i}^{2} .
$$

We are now ready to match up the data developed in the line search, and summarized in (2.6), with the local approximation (3.5). We shall require that:

$$
R_{i}\left(\alpha_{i}^{(1)}\right)=F_{i}\left(\alpha_{i}^{(1)}\right), \quad R_{i}\left(\alpha_{i}^{(2)}\right)=F_{i}\left(\alpha_{i}^{(2)}\right), \quad R_{i}\left(\alpha_{i}^{(3)}\right)=F_{i}\left(\alpha_{i}^{(3)}\right) .
$$

More explicitly, Eqs. (3.6) are:

$$
\begin{aligned}
& a_{i}+b_{i} \alpha_{i}^{(1)}+1 / 2 c_{i}\left(\alpha_{i}^{(1)}\right)^{2}=F_{i}\left(\alpha_{i}^{(1)}\right), \\
& a_{i}+b_{i} \alpha_{i}^{(2)}+1 / 2 c_{i}\left(\alpha_{i}^{(2)}\right)^{2}=F_{i}\left(\alpha_{i}^{(2)}\right), \\
& a_{i}+b_{i} \alpha_{i}^{(3)}+1 / 2 c_{i}\left(\alpha_{i}^{(3)}\right)^{2}=F_{i}\left(\alpha_{i}^{(3)}\right),
\end{aligned}
$$

which can be solved for $a_{i}, b_{i}$ and $c_{i}$ in terms of the known quantities

$$
\left\{\alpha_{i}^{(1)}, \alpha_{i}^{(2)}, \alpha_{i}^{(3)}\right\} \quad \text { and } \quad\left\{F_{i}\left(\alpha_{i}^{(1)}\right), F_{i}\left(\alpha_{i}^{(2)}\right), F_{i}\left(\alpha_{i}^{(3)}\right)\right\}
$$

We may now regard the data gleaned in each line search as summarized implicitly in the calculated values of $a_{i}, b_{i}$ and $c_{i}{ }^{4}$ Referring back to their definitions, we may write: ${ }^{5}$

$$
\begin{gathered}
s_{i}^{T} g_{0}^{*}+s_{i}^{T} G^{*} \tau_{i-1}=b_{i}, \\
s_{i}^{T} G^{*} s_{i}=c_{i} ;
\end{gathered}
$$

and we have thus generated conditions on $g_{0}^{*}$ and $G^{*}$ in terms of the known quantities $\left\{s_{i}, \tau_{i}, b_{i}, c_{i}\right\}$. These conditions hold for $i=1, \ldots, N$, i.e., for every step in the cycle.

We now introduce additive corrections to $g_{0}$ and $G$, defined as follows:

$$
\begin{aligned}
g_{0}^{*} & \equiv g_{0}+\gamma, \\
G^{*} & \equiv G+\Gamma .
\end{aligned}
$$

Equations (3.8) can then be rewritten in terms of the new unknowns $\gamma$ and $\Gamma$ :

$$
s_{i}^{T} \gamma+s_{i}^{T} \Gamma \tau_{i-1}=b_{i}-s_{i}^{T} g_{0}-s_{i}^{T} G \tau_{i-1} \equiv \epsilon_{i},
$$

\footnotetext{
${ }^{4}$ Because of (2.6) it may readily be proved that $c_{i}>0$.

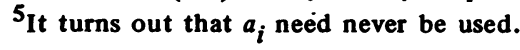




$$
s_{i}^{T} \Gamma s_{i}=c_{i}-s_{i}^{T} G s_{i}=c_{i}-1 .
$$

The last reduction follows from (2.11). We can also reduce (3.10a) by noting that, based on (2.2) and (2.3):

$$
\tau_{i}=\tau_{i-1}+h_{i} s_{i}
$$

which implies that

$$
\tau_{i}=\sum_{j=1}^{i} h_{j} s_{j}
$$

Since $\tau_{i-1}$ clearly does not include $s_{i}$, and since $s_{i}$ is conjugate to all $\left\{s_{j}\right\}$ with $j<i$, we have:

$$
s_{i}^{T} G \tau_{i-1}=0
$$

from which it follows that $\epsilon_{i}$ can be reduced, so that (3.10a) becomes:

$$
s_{i}^{T} \gamma+s_{i}^{T} \Gamma \tau_{i-1}=\epsilon_{i}=b_{i}-s_{i}^{T} g_{0} .
$$

Equations (3.10b) and (3.14) are the QN conditions for this problem.

4. Variational Derivation of $\Gamma$. After having completed a cycle of $N$ steps, we consider next how to use the information collected to estimate the corrections $\gamma$ and $\Gamma$. In [1], a functional was constructed, involving both quantities; and a variational procedure was used to derive formulas for both. However, there were serious ambiguities in that approach, ${ }^{6}$ so that we shall now depart from that scheme.

Our strategy will be to regard $\gamma$ as merely a (vector) parameter, and to concentrate at first on $\Gamma$ alone. If $G$ (and hence $\Gamma$ ) be regarded as a covariant tensor of second rank (as it is when thought of as a "metric"), then the simplest quadratic invariant involving $\Gamma$ would be (with a convenience factor of $1 / 2$ ):

$$
\Phi_{0} \equiv \not / 2 \operatorname{Tr}\left\{G^{-1} \Gamma G^{-1} \Gamma^{T}\right\}
$$

(where the symbol Tr indicates the trace). We are not assuming $\Gamma$ to be symmetric a priori, but will require it to come out that way.

To the bare functional $\Phi_{0}$, we must adjoin the QN constraints, as well as the symmetry constraint on $\Gamma$. We use the Lagrange multipliers $\left\{\theta_{i}\right\},\left\{\eta_{i}\right\}$ and $\Lambda$ (a matrix). The complete functional is then:

$$
\begin{aligned}
\Phi= & 1 / 2 \operatorname{Tr}\left\{G^{-1} \Gamma G^{-1} \Gamma^{T}\right\}-2 \sum_{i=1}^{N} \theta_{i}\left\{s_{i}^{T}\left(\gamma+\Gamma \tau_{i-1}\right)-\epsilon_{i}\right\} \\
& -\sum_{i=1}^{N} \eta_{i}\left\{s_{i}^{T} \Gamma s_{i}-c_{i}+1\right\}-\operatorname{Tr}\left\{\Lambda\left(\Gamma-\Gamma^{T}\right)\right\} .
\end{aligned}
$$

We follow the method of solution described in [1], but shall not go into detail here; the formula for $\Gamma$ turns out to be: 


$$
\Gamma=G \sum_{i=1}^{N}\left\{\theta_{i}\left(s_{i} \tau_{i-1}^{T}+\tau_{i-1} s_{i}^{T}\right)+\eta_{i} s_{i} s_{i}^{T}\right\} G
$$

(Note that, although $\theta_{1}$ appears formally, it is not really included, because of the vanishing of $\tau_{0}$.)

The $\eta$ 's may be immediately evaluated by applying QN condition (3.10b). We have:

$$
\begin{aligned}
s_{k}^{T} \Gamma s_{k} & =\sum_{i=1}^{N}\left\{\theta_{i}\left[s_{k}^{T} G s_{i} \tau_{i-1}^{T} G s_{k}+s_{k}^{T} G \tau_{i-1} s_{i}^{T} G s_{k}\right]+\eta_{i} s_{k}^{T} G s_{i} s_{i}^{T} G s_{k}\right\} \\
& =\sum_{i=1}^{N}\left\{\theta_{i}\left[\delta_{i k} \tau_{i-1}^{T} G s_{k}+\delta_{i k} s_{k}^{T} G \tau_{i-1}\right]+\eta_{i} \delta_{k i} \delta_{i k}\right\} \\
& =2 \theta_{k} \tau_{k-1}^{T} G s_{k}+\eta_{k}=\eta_{k}=c_{k}-1 .
\end{aligned}
$$

The various reductions follow from (2.11) and (3.13).

We next apply the remaining QN condition (3.14) to $\Gamma$ and $\gamma$. Substituting for $\Gamma$ from (4.3), we obtain:

$$
\begin{aligned}
s_{i}^{T} \Gamma \tau_{i-1} & =s_{i} G \sum_{j}\left\{\theta_{j}\left(s_{j} \tau_{j-1}^{T}+\tau_{j-1} s_{j}^{T}\right)+\eta_{j} s_{j} s_{j}^{T}\right\} G \tau_{i-1} \\
& =\sum_{j}\left\{\theta_{j}\left(\delta_{i j} \tau_{j-1}^{T} G \tau_{i-1}+s_{i}^{T} G \tau_{j-1} s_{i}^{T} G \tau_{i-1}\right)+\eta_{j} \delta_{i j} s_{j}^{T} G \tau_{i-1}\right\} \\
& =\theta_{i} \tau_{i-1}^{T} G \tau_{i-1}+\sum_{i} \theta_{j}\left(s_{i}^{T} G \tau_{j-1}\right)\left(s_{j}^{T} G \tau_{i-1}\right)+\eta_{i} s_{i}^{T} G \tau_{i-1}
\end{aligned}
$$

The last term above vanishes because of (3.13). The term preceding that vanishes too because regardless of the values of $i$ and $j$, at least one of the factors is zero (again because of the conjugacy of the $\left.\left\{s_{i}\right\}\right)$. If we define:

$$
\tau_{i-1}^{2} \equiv \tau_{i-1}^{T} G \tau_{i-1},
$$

then we can write

$$
s_{i} \Gamma \tau_{i-1}=\theta_{i} \tau_{i-1}^{2}
$$

We can greatly simplify (3.14), if we recall that the set of vectors $\left\{G s_{i}\right\}$ is complete. This means that we can expand the vector $\gamma$ as follows:

$$
\gamma=\sum_{j=1}^{N} \mu_{j} G s_{j}
$$

so that

$$
s_{i}^{T} \gamma=\sum_{j=1}^{N} \mu_{j} \delta_{i j}=\mu_{i}
$$

and (3.14) reduces to:

$$
\mu_{i}+\tau_{i-1}^{2} \theta_{i}=\epsilon_{i}
$$


Since the $\left\{c_{i}\right\}$ are known quantities, we need not concern ourselves further with the QN condition (4.4). On the other hand, the QN condition (4.10) involves two unknown quantities (viz., $\mu_{i}$ and $\theta_{i}$ ) for each step. As we shall see, the constraints on $\left\{\theta_{i}\right\}$ which are necessary to insure the positive-definiteness of $G^{*}$ will enable us to determine both quantities.

5. Maintenance of Positive-Definiteness. We shall first express $G^{*}$ directly in terms of $G$ by applying the correction (3.9b) explicitly. We obtain, with the help of (4.3), (4.4) and (2.16):

$$
\begin{aligned}
G^{*} & =G+\Gamma=\sum_{i=1}^{N} G s_{i} s_{i}^{T} G+\Gamma \\
& =\sum_{i=1}^{N} G s_{i} s_{i}^{T} G+\sum_{i=1}^{N}\left(c_{i}-1\right) G s_{i} s_{i}^{T} G+\sum_{i=1}^{N} \theta_{i} G\left(s_{i} \tau_{i-1}^{T}+\tau_{i-1} s_{i}^{T}\right) G \\
& =\sum_{i=1}^{N}\left\{c_{i} G s_{i} s_{i}^{T} G+\tau_{i} G\left(s_{i} \tau_{i-1}^{T}+\tau_{i-1} s_{i}^{T}\right) G\right\} .
\end{aligned}
$$

Our subsequent analysis will be greatly simplified if we transform $G^{*}$ as follows, to form $B$ :

$$
B \equiv S^{T} G^{*} S,
$$

where $S$ is defined as in (2.12). The elements of $B$ are given by:

$$
\begin{aligned}
B_{k m} & =s_{k}^{T} G^{*} s_{m} \\
& =s_{k}^{T} \sum_{i=1}^{N}\left\{c_{i} G s_{i} s_{i}^{T} G+\theta_{i} G\left(s_{i} \tau_{i-1}^{T}+\tau_{i-1} s_{i}^{T}\right) G\right\} s_{m} \\
& =\sum_{i=1}^{N}\left\{c_{i}\left(s_{k}^{T} G s_{i}\right)\left(s_{i}^{T} G s_{m}\right)+\theta_{i}\left[\left(s_{k}^{T} G s_{i}\right)\left(\tau_{i-1}^{T} G s_{m}\right)+\left(s_{k}^{T} G \tau_{i-1}\right)\left(s_{i}^{T} G s_{m}\right)\right]\right\} \\
& =\sum_{i=1}^{N}\left\{c_{i} \delta_{k i} \delta_{i m}+\theta_{i}\left[\delta_{k i}\left(\tau_{i-1}^{T} G s_{m}\right)+\left(s_{k}^{T} G \tau_{i-1}\right) \delta_{i m}\right]\right\} \\
& =c_{k} \delta_{k m}+\theta_{k}\left(\tau_{k-1}^{T} G s_{m}\right)+\theta_{m}\left(s_{k}^{T} G \tau_{m-1}\right) .
\end{aligned}
$$

The reductions are based on the conjugacy relation (2.11). Further simplification may be effected by generalizing (3.13), based on the expression (3.12) for $\tau_{i}$. Since $\left(\tau_{k-1}^{T} G s_{m}\right)$ is the same as $\left(s_{m}^{T} G \tau_{k-1}\right)$, we need consider only the latter. Clearly, if $k \leqslant m$, this expression vanishes, since $\tau_{k-1}$ does not then contain $s_{m}$. On the other hand, if $k>m$, then the surviving part of the inner product is $h_{m}\left(s_{m}^{T} G s_{m}\right)$ which, of course, is just equal to $h_{m}$. We can summarize as follows:

$$
\begin{array}{rlrl}
\left(s_{m}^{T} G \tau_{k-1}\right) & =0 & & \text { if } k \leqslant m, \\
& =h_{m} & \text { if } k>m .
\end{array}
$$


On this basis, we can display $B$ :

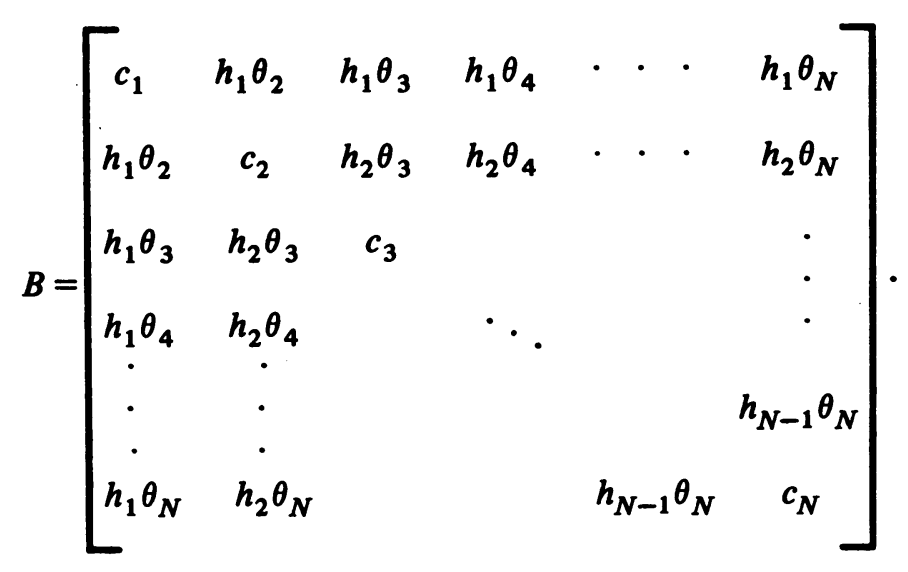

Equation (5.2) can be solved for $G^{*}$ by multiplying by $R$ and using (2.14). We have:

$$
R B R^{T}=\left(R S^{T}\right) G^{*}\left(S R^{T}\right)=G^{*}
$$

which shows, together with (5.2), that $G^{*}$ will be positive-definite if and only if $B$ is. We may, therefore, concentrate our efforts on $B$.

There are undoubtedly several ways of accomplishing our end; we shall consider two, but display numerical results for only one of them.

First, we shall concentrate on keeping all the eigenvalues of $B$ positive. This may be done by the use of Gershgorin's Theorem [2]. If $\lambda$ is any eigenvalue of $B$, then it satisfies:

$$
\left|\lambda-B_{i i}\right| \leqslant \sum_{j \neq i}\left|B_{i j}\right|
$$

which means that

$$
\lambda \geqslant B_{i i}-\sum_{j \neq i}\left|B_{i j}\right|
$$

so that if, for some number $\phi_{i}>0$, we insure that

$$
B_{i i}-\sum_{j \neq i}\left|B_{i j}\right| \geqslant \phi_{i}
$$

we then have

$$
\lambda \geqslant \min _{i} \phi_{i}>0
$$

From (5.5), it is clear that:

$$
\begin{gathered}
B_{i i}=c_{i}, \\
\sum_{j \neq i}\left|B_{i j}\right|=\left|\theta_{i}\right| \sum_{j=1}^{i-1} h_{j}+h_{i} \sum_{j=i+1}^{N}\left|\theta_{j}\right| .
\end{gathered}
$$

Since, as was indicated previously, all of the $\left\{c_{i}\right\}$ are positive, we may "scale" the $\phi_{i}$, in a sense, by setting:

$$
\phi_{i}=\beta_{i} c_{i}
$$


where $\beta_{i}>0$. Substituting these relations in (5.9), we ask that:

$$
c_{i}-\left|\theta_{i}\right| \sum_{j=1}^{i-1} h_{j}-h_{i} \sum_{j=i+1}^{N}\left|\theta_{j}\right| \geqslant \beta_{i} c_{i},
$$

in which case, we shall have:

$$
\lambda \geqslant \min _{i} \beta_{i} c_{i}
$$

If we rewrite (5.13), we obtain:

$$
\left(\sum_{j=1}^{i-1} h_{j}\right)\left|\theta_{i}\right| \leqslant\left(1-\beta_{i}\right) c_{i}-h_{i} \cdot \sum_{j=i+1}^{N}\left|\theta_{j}\right|
$$

which for $i=1, \ldots, N$ serves as a set of bounds on $\left\{\left|\theta_{i}\right|\right\}$. (Clearly, $\beta_{i}$ must be less than unity.) These bounds may be applied recursively, starting with $\theta_{N}$. Thus, for example:

$$
\left(\sum_{j=1}^{N-1} h_{j}\right)\left|\theta_{N}\right| \leqslant\left(1-\beta_{N}\right) c_{N}
$$

$$
\left(\sum_{j=1}^{N-2} h_{j}\right)\left|\theta_{N-1}\right| \leqslant\left(1-\beta_{N-1}\right) c_{N-1}-h_{N-1}\left|\theta_{N}\right|
$$

etc.

We shall next consider another method ${ }^{7}$ for bounding the $\theta$ 's, related not to the eigenvalues of $B$, but to a sequence of principal minors of $B$.

If we define the matrix $B_{i}$ as follows:

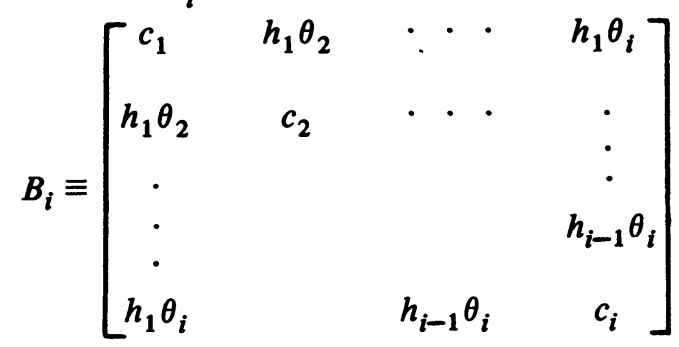

and the vector $q_{i}$ by:

$$
q_{i} \equiv\left\{h_{1}, h_{2}, \ldots, h_{i}\right\}
$$

then, clearly, we have the recursion:

$$
B_{i}=\left[\begin{array}{cc}
B_{i-1} & q_{i-1} \theta_{i} \\
q_{i-1}^{T} \theta_{i} & c_{i}
\end{array}\right]
$$

and we shall attempt to insure the positive-definiteness of $B_{i}$, given that of $B_{i-1}$. If this can be done for all $i$ then, since $B_{N}=B$, we shall have our result.

To further facilitate the analysis, we transform $B_{i}$ with the matrix $Q_{i}$, defined by:

${ }^{7}$ Which is based on a suggestion made by Dr. S. Schechter. 


$$
Q_{i} \equiv\left[\begin{array}{cc}
I_{i-1} & -\theta_{i} B_{i-1}^{-1} q_{i-1} \\
0 & 1
\end{array}\right]
$$

to obtain the new matrix $D_{i}$ :

$$
D_{i} \equiv Q_{i}^{T} B_{i} Q_{i}=\left[\begin{array}{cc}
B_{i-1} & 0 \\
0 & \phi_{i}
\end{array}\right],
$$

where

$$
\phi_{i} \equiv c_{i}-\left(q_{i-1}^{T} B_{i-1}^{-1} q_{i-1}\right) \theta_{i}^{2} .
$$

As before, if $D_{i}$ can be kept positive-definite, then $B_{i}$ will be also. Since $B_{i-1}$ has been assumed to be positive-definite, then $D_{i}$ is positive-definite if and only if $\phi_{i}>0$. We therefore choose some positive number $\beta_{i}$, and require that:

$$
\phi_{i} \equiv c_{i}-\omega_{i-1} \theta_{i}^{2} \geqslant \beta_{i} c_{i}>0,
$$

where

$$
\omega_{i} \equiv q_{i}^{T} B_{i}^{-1} q_{i},
$$

and this in turn establishes the constraint on $\theta_{i}$ :

$$
\omega_{i-1} \theta_{i}^{2} \leqslant\left(1-\beta_{i}\right) c_{i}
$$

(and again, $\beta_{i}$ must be less than unity).

If this recursive process is continued until $i=N$, we then have a positive-definite $D_{N}$; hence a positive-definite $B_{N}$; hence a positive-definite $G^{*}$.

6. Selection of $\theta_{i}$ and $\mu_{i}$. The remaining QN condition, Eq. (4.10) will now be used in conjunction with the constraints on $\left\{\theta_{i}\right\}$, to effect unique choices for $\theta_{i}$ and $\mu_{i}$ at each step. Clearly, for $i=1$, we have the forced choice:

$$
\mu_{1}=\epsilon_{1}
$$

and, as remarked previously, $\theta_{1}$ does not enter into the problem at all.

For $i>1$, our strategy will be to choose the $\mu_{i}$ of smallest magnitude, consistent with the constraint on $\theta_{i}$. This strategy is in the same spirit of "minimal correction" which prompted the formulation of the selection of $\Gamma$ as a variational problem.

If there were no constraints on the $\theta$ 's, the choice would obviously be

$$
\left.\begin{array}{l}
\mu_{i}=0 \\
\theta_{i}=\epsilon_{i} / \tau_{i-1}^{2}
\end{array}\right\}, \quad i>1 .
$$

However, this strategy almost always leads to an indefinite $G^{*}$, with catastrophic results (as observed in practice). This is the reason for applying the constraints to keep $G^{*}$ positive-definite. 


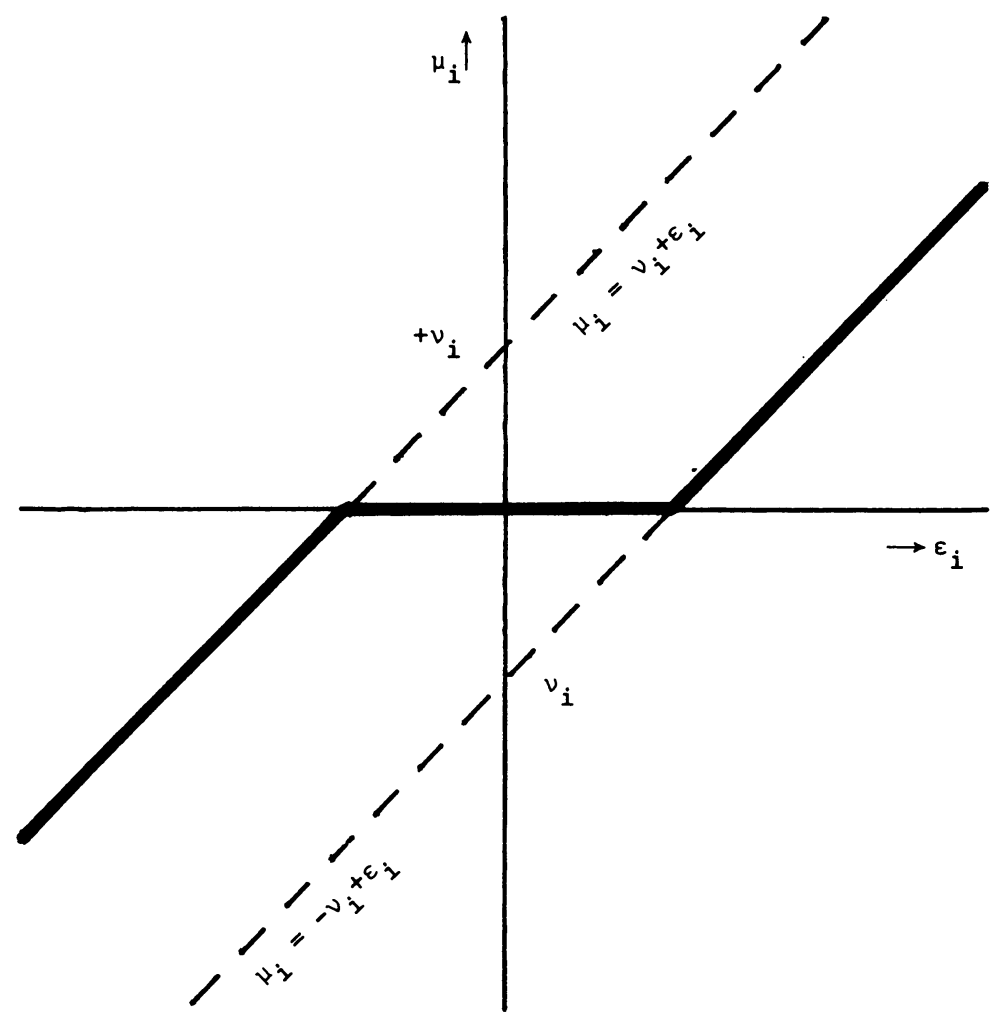

FIGURE 1

Since the constraints (5.15) and (5.25) may both be written in the same form:

$$
\left|\theta_{i}\right| \leqslant \lambda_{i}
$$

with $\lambda_{i}>0$, we shall treat them together. From (4.10) we have:

$$
\epsilon_{i}-\mu_{i}=\tau_{i-1}^{2} \theta_{i}
$$

so that

$$
\left|\epsilon_{i}-\mu_{i}\right|=\tau_{i-1}^{2}\left|\theta_{i}\right| \leqslant \tau_{i-1}^{2} \lambda_{i} \equiv \nu_{i} .
$$

We now wish to choose $\mu_{i}$ as small as possible in magnitude consistent with (6.5). This is a (trivial) linear programming problem, which may be solved graphically. In Figure 1, the two oblique lines bound the region of the $(\epsilon, \mu)$ plane wherein $(6.5)$ is satisfied. The heavy line traces the minimum magnitude $\mu_{i}$ within this region. This solution may be written as:

$$
\mu_{i}=\operatorname{sign}\left(\epsilon_{i}\right) \times \max \left(0,\left|\epsilon_{i}\right|-\nu_{i}\right)
$$

with this choice of $\mu_{i}, \theta_{i}$ may now be determined from (6.4). In this way, we have, so to speak, "apportioned" the increments to the corrections $\Gamma$ and $\gamma$ in a natural manner by using the constraints on $G^{*}$. 
Thus, at the end of a cycle, we are in a position to update $g_{0}$ and $G$, according to (3.9), (4.3) and (4.8). In addition, because $g_{0}$ is assumed to vary linearly with $x$, we must perform a translation of it, to the new starting point. If we denote the translated value by $g_{0}^{* *}$, we have

$$
g_{0}^{* *}=g_{0}^{*}+G^{*} \tau_{N}
$$

7. Choice of $\left\{\beta_{i}\right\}$ in Second Method. As a matter of experience the second method described in Section 5 for maintaining positive-definiteness turned out to be considerably the better. Hence, all of our results are for this method.

The choice of the $\beta$ 's remains arbitrary. By way of a guide, we shall examine the effect of the $\beta$-values on the determinant of $G^{*}$. We have:

$$
\operatorname{det} G^{*}=\left(\operatorname{det} G^{*}\right)(\operatorname{det} G)^{-1}(\operatorname{det} G)=\operatorname{det}\left(G^{*} G^{-1}\right)(\operatorname{det} G)
$$

and, using (5.6):

(7.2) $\operatorname{det}\left(G^{*} G^{-1}\right)=\operatorname{det}\left(R B R^{T} G^{-1}\right)=\operatorname{det}\left(B R^{T} G^{-1} R\right)=\operatorname{det}(B) \operatorname{det}\left(R^{T} G^{-1} R\right)$.

But, from (2.15) and (2.14):

$$
\operatorname{det}\left(R^{T} G^{-1} R\right)=\operatorname{det}\left(S^{-1} G^{-1} R\right)=\operatorname{det}\left[(G S)^{-1} R\right]=\operatorname{det}\left[R^{-1} R\right]=1
$$

so that, finally:

$$
\operatorname{det} G^{*}=(\operatorname{det} B)(\operatorname{det} G)
$$

Next, from (5.21), we have:

$$
\operatorname{det} D_{i}=\operatorname{det}\left(Q_{i}^{T} B_{i} Q_{i}\right)=\left(\operatorname{det} B_{i}\right) \times\left(\operatorname{det} Q_{i}\right)^{2} ;
$$

but it is clear from the form (5.20) of $Q_{i}$ that $\operatorname{det} Q_{i}=1$, so that

$$
\operatorname{det} B_{i}=\operatorname{det} D_{i} \text {. }
$$

On the other hand, it is also clear from (5.21) that

$$
\operatorname{det} B_{i}=\operatorname{det} D_{i}=\left(\operatorname{det} B_{i-1}\right) \times \phi_{i}
$$

which gives a recursion for $\operatorname{det} B_{i}$, and since $B_{1}=\phi_{1}$, we conclude that:

$$
\operatorname{det} B=\prod_{i=1}^{N} \phi_{i} \text {. }
$$

Using all these results, together with the constraints (5.23), we can bound $\operatorname{det} G^{*}$ below as follows:

$$
\begin{aligned}
\operatorname{det} G^{*} & =(\operatorname{det} G) \times(\operatorname{det} B)=\operatorname{det} G \times\left(\prod_{i} \phi_{i}\right) \\
& \geqslant \operatorname{det} G \times\left(\prod_{i} c_{i}\right) \times\left(\prod_{i} \beta_{i}\right) .
\end{aligned}
$$

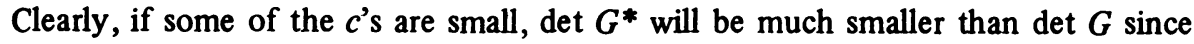
$\beta_{i}<1$. Whatever the case, it is obviously advantageous to try to keep the determinants as large as possible. This means that the $\beta$ 's should be fairly close to unity. 
The most obvious way of "balancing" the $\beta$ 's is to set them all equal to a predetermined constant. We may simply choose a value, once and for all, or make use of (7.9) as a guide to fitting a value to each problem. If all of the $\beta$ 's are equal; (7.9) becomes:

$$
\operatorname{det} G^{*} \geqslant\left[(\operatorname{det} G) \times\left(\prod_{i} c_{i}\right)\right] \times \beta^{N}
$$

Since we have no control over the factor within the brackets, we can ignore it, and concentrate our attention on $\beta^{N}$. If we demand that this factor should be no less than some fixed constant $\rho$, then we should set $\beta^{N}=\rho$, so that:

$$
\beta=\rho^{1 / N},
$$

which has the desirable property that $\beta$ gets closer and closer to unity as $N$ gets larger. The value of $\rho$ must be established by numerical experiment.

8. Numerical Results. We have performed our tests on many of the "standard" functions in the literature using the "standard" starting points. We list the names of these functions here, with appropriate references, and add any comments that serve to clarify our results ( $N$ is the number of arguments):

(1) Helical Valley [3]

(2) Rosenbrock's Function [4].

(3) Wood's Function [5].

(4) Powell's Quartic Function [6].

(5) Watson's Function [7]. This has been tested for $N=6$ and 9.

(6) Chebyquad [8]. This has been tested for $N=4,6,8$ and 20.

(7) Random Trigonometric Functions [3]. These are trigonometric polynomials whose coefficients are random variables (fixed, of course, for each case). The starting points are also random variables. Because of this, the behavior of each function so generated is unique and unpredictable, so that 3 runs were made for each case. Runs were done for $N=3,5,10$, and 20 , and the number of function evaluations averaged. Those runs wherein the method converged ${ }^{8}$ to a minimum different from the predetermined one were ignored, since they do not support a fair comparison. All the runs shown to converge did so to the correct solutions.

(8) Biggs' Exponential Functions [9]. There are two functions, called EXP5 and EXP6 with 5 and 6 arguments, respectively.

In Table 1 are shown the numbers of function evaluations necessary for convergence for most of these functions, when the value $\beta$ is fixed independently of $N$. Since $0<\beta<1$, the five $\beta$-values covering this range were tried. It is abundantly clear that, although fixing $\beta$ may be satisfactory when $N$ (indicated in parentheses) is small, it is totally unsatisfactory for large $N$, as evidenced by the failures of convergence (marked "F") for Chebyquad and the trigonometric functions when $N=20$.

${ }^{8}$ For all functions but the random trigonometric functions, convergence was defined as requiring that $g^{T} G^{-1}<10^{-12}$. For the trigonometric functions, it was defined as requiring that $\max _{i}\left(\left|x_{i}-x_{0 i}\right|\right)<10^{-6}$, where $x_{0}$ was the known location of the correct minimum. 


\section{TABLE 1}

Function evaluations vs $\beta$-values

\begin{tabular}{|c|c|c|c|c|c|}
\hline & .1 & .3 & .5 & .7 & .9 \\
\hline \multicolumn{6}{|l|}{ Function } \\
\hline $\begin{array}{ll}\text { Beale } & (2) \\
\text { Hel } & (3) \\
\text { Ros } & (2) \\
\text { Wood } & (4) \\
\text { Pow } & (4) \\
\text { Wat } & (6)\end{array}$ & $\begin{array}{r}85 \\
285 \\
236 \\
508 \\
1060 \\
940\end{array}$ & $\begin{array}{r}76 \\
287 \\
174 \\
316 \\
668 \\
1064\end{array}$ & $\begin{array}{r}86 \\
315 \\
203 \\
314 \\
568 \\
662\end{array}$ & $\begin{array}{r}66 \\
232 \\
203 \\
266 \\
467 \\
456\end{array}$ & $\begin{array}{r}82 \\
239 \\
165 \\
288 \\
711 \\
540\end{array}$ \\
\hline $\begin{array}{r}\text { Cheb }(4) \\
(6) \\
(8) \\
(20)\end{array}$ & $\begin{array}{r}222 \\
343 \\
3780 \\
F\end{array}$ & $\begin{array}{c}176 \\
403 \\
1524 \\
F\end{array}$ & $\begin{array}{c}326 \\
314 \\
684 \\
F\end{array}$ & $\begin{array}{r}122 \\
346 \\
836 \\
5044\end{array}$ & $\begin{array}{r}138 \\
253 \\
603 \\
3499\end{array}$ \\
\hline $\begin{array}{c}\text { Trig } \quad(3) \\
" 1\end{array}$ & $\begin{array}{c}F \\
64 \\
120 \\
\end{array}$ & $\begin{array}{r}85 \\
116 \\
113 \\
\end{array}$ & $\begin{array}{r}126 \\
85 \\
74 \\
\end{array}$ & $\begin{array}{l}122 \\
102 \\
105 \\
\end{array}$ & $\begin{array}{r}82 \\
118 \\
148 \\
\end{array}$ \\
\hline MEAN & $\mathbf{F}$ & 105 & 95 & 110 & 116 \\
\hline $\begin{array}{c}\text { Trig (5) } \\
" 1 \\
\end{array}$ & $\begin{array}{c}737 \\
F \\
430 \\
\end{array}$ & $\begin{array}{l}244 \\
235 \\
303 \\
\end{array}$ & $\begin{array}{l}249 \\
260 \\
225 \\
\end{array}$ & $\begin{array}{l}215 \\
218 \\
331 \\
\end{array}$ & $\begin{array}{l}158 \\
220 \\
180 \\
\end{array}$ \\
\hline MEAN & $F$ & 261 & 245 & 255 & 186 \\
\hline $\begin{array}{c}\operatorname{Trig}(10) \\
"\end{array}$ & $\begin{array}{r}F \\
F \\
22246 \\
\end{array}$ & $\begin{array}{l}3336 \\
3681 \\
2548 \\
\end{array}$ & $\begin{array}{r}743 \\
1422 \\
1721 \\
\end{array}$ & $\begin{array}{l}874 \\
512 \\
644 \\
\end{array}$ & $\begin{array}{l}978 \\
728 \\
610 \\
\end{array}$ \\
\hline MEAN & $\mathbf{F}$ & 3188 & 1295 & 677 & 772 \\
\hline $\begin{array}{r}\text { Trig }(20) \\
" 1 \\
\end{array}$ & $\begin{array}{l}F \\
F \\
F\end{array}$ & $\begin{array}{l}\mathrm{F} \\
\mathrm{F} \\
\mathrm{F}\end{array}$ & $\begin{array}{l}\mathrm{F} \\
\mathrm{F} \\
\mathrm{F}\end{array}$ & $\begin{array}{l}3611 \\
3988 \\
3471 \\
\end{array}$ & $\begin{array}{l}1949 \\
2789 \\
2740 \\
\end{array}$ \\
\hline MEAN & $\mathbf{F}$ & F & $F$ & 3690 & 2493 \\
\hline
\end{tabular}

The results with $\beta$ determined from Eq. (7.11) are shown in Table 2 for nine representative values of $\rho$ over its allowable range. Clearly, the performance is far better (since there are no failures) and the performance of the algorithm is relatively insensitive to the $\rho$-values. However, the value $\rho=.5$ seems slightly better than the others, so that this value was used for further runs.

For comparison with the results of Gill, Murray and Pitfield [10] (GMP) the convergence criterion was adjusted for each function, for termination when the difference between the function value at the end of a cycle and its known minimum value fell within the accuracy given by GMP. In Table 3 are shown the numbers of cycles (noted as ITER), the number of function evaluations (EVALS), and the final accuracy (ACCUR.). The DFQN method is comparable to GMP except for the Chebyquad cases, EXP5 and EXP6. The reason for this poor behavior is not known. (The $\mathrm{L}$ in the last line indicates that a local minimum was found.)

In Table 4, the DFQN method applied to the random trigonometric functions is compared with the results quoted by Powell [11] for his 1964 method requiring no derivatives. As can be seen, the DFQN method is slightly worse, but manages to keep up for large $N$. An additional set of three cases for $N=50$ was run, with the 
TABLE 2

Function evaluations vs $\rho$-values

\begin{tabular}{|c|c|c|c|c|c|c|c|c|c|c|}
\hline & $\rho \rightarrow$ & .1 & .2 & .3 & .4 & .5 & .6 & .7 & .8 & .9 \\
\hline \multicolumn{2}{|c|}{ Function } & & & & & & & & & \\
\hline $\begin{array}{l}\text { Beale } \\
\text { Hel } \\
\text { Ros } \\
\text { Wood } \\
\text { Pow } \\
\text { Wat }\end{array}$ & $\begin{array}{l}(2) \\
(3) \\
(2) \\
(4) \\
(4) \\
(6)\end{array}$ & $\begin{array}{r}78 \\
251 \\
189 \\
287 \\
634 \\
476\end{array}$ & $\begin{array}{r}91 \\
269 \\
220 \\
277 \\
806 \\
582\end{array}$ & $\begin{array}{r}81 \\
250 \\
146 \\
331 \\
556 \\
385\end{array}$ & $\begin{array}{r}68 \\
202 \\
147 \\
301 \\
674 \\
371\end{array}$ & $\begin{array}{r}74 \\
194 \\
144 \\
277 \\
501 \\
369\end{array}$ & $\begin{array}{r}66 \\
204 \\
137 \\
261 \\
576 \\
354\end{array}$ & $\begin{array}{r}85 \\
255 \\
163 \\
257 \\
636 \\
346\end{array}$ & $\begin{array}{r}88 \\
285 \\
175 \\
243 \\
561 \\
386\end{array}$ & $\begin{array}{r}63 \\
282 \\
148 \\
228 \\
527 \\
478\end{array}$ \\
\hline Cheb & $\begin{array}{r}(4) \\
(6) \\
(8) \\
(20)\end{array}$ & $\begin{array}{r}228 \\
273 \\
563 \\
3454\end{array}$ & $\begin{array}{r}122 \\
341 \\
503 \\
3155\end{array}$ & $\begin{array}{r}122 \\
359 \\
567 \\
3053\end{array}$ & $\begin{array}{r}129 \\
426 \\
744 \\
2963\end{array}$ & $\begin{array}{r}121 \\
268 \\
503 \\
3069\end{array}$ & $\begin{array}{r}121 \\
328 \\
578 \\
2820\end{array}$ & $\begin{array}{r}130 \\
329 \\
593 \\
3023\end{array}$ & $\begin{array}{r}130 \\
360 \\
587 \\
3099\end{array}$ & $\begin{array}{r}134 \\
366 \\
635 \\
3253\end{array}$ \\
\hline Trig & $\begin{array}{c}(3) \\
" \\
"\end{array}$ & $\begin{array}{l}78 \\
73 \\
77 \\
\end{array}$ & $\begin{array}{l}63 \\
77 \\
71 \\
\end{array}$ & $\begin{array}{l}96 \\
60 \\
59 \\
\end{array}$ & $\begin{array}{l}90 \\
76 \\
74 \\
\end{array}$ & $\begin{array}{l}78 \\
68 \\
71 \\
\end{array}$ & $\begin{array}{r}66 \\
121 \\
132 \\
\end{array}$ & $\begin{array}{r}104 \\
63 \\
93 \\
\end{array}$ & $\begin{array}{r}75 \\
74 \\
107 \\
\end{array}$ & $\begin{array}{r}92 \\
108 \\
115 \\
\end{array}$ \\
\hline MEAN & & 76 & 70 & 72 & 70 & 72 & 68 & 87 & 85 & 105 \\
\hline Trig & $\begin{array}{c}(5) \\
" \\
"\end{array}$ & $\begin{array}{l}219 \\
294 \\
187 \\
\end{array}$ & $\begin{array}{l}204 \\
181 \\
207 \\
\end{array}$ & $\begin{array}{l}179 \\
176 \\
216 \\
\end{array}$ & $\begin{array}{l}212 \\
178 \\
517 \\
\end{array}$ & $\begin{array}{l}183 \\
183 \\
207 \\
\end{array}$ & $\begin{array}{l}282 \\
202 \\
178 \\
\end{array}$ & $\begin{array}{l}267 \\
199 \\
214 \\
\end{array}$ & $\begin{array}{l}259 \\
242 \\
224 \\
\end{array}$ & $\begin{array}{l}155 \\
224 \\
189 \\
\end{array}$ \\
\hline MEAN & & 233 & 197 & 190 & 302 & 191 & 221 & 227 & 242 & 189 \\
\hline Trig & $\begin{array}{c}(10) \\
" \\
"\end{array}$ & $\begin{array}{l}822 \\
778 \\
598 \\
\end{array}$ & $\begin{array}{l}538 \\
606 \\
566 \\
\end{array}$ & $\begin{array}{l}577 \\
715 \\
753 \\
\end{array}$ & $\begin{array}{l}617 \\
674 \\
636 \\
\end{array}$ & $\begin{array}{l}664 \\
626 \\
768 \\
\end{array}$ & $\begin{array}{l}754 \\
928 \\
696 \\
\end{array}$ & $\begin{array}{l}933 \\
863 \\
617 \\
\end{array}$ & $\begin{array}{l}579 \\
727 \\
841 \\
\end{array}$ & $\begin{array}{l}708 \\
612 \\
751 \\
\end{array}$ \\
\hline MEAN & & 733 & 570 & 682 & 642 & 686 & 793 & 804 & 716 & 690 \\
\hline Trig & $\begin{array}{c}(20) \\
" \\
"\end{array}$ & $\begin{array}{l}2061 \\
2428 \\
2651 \\
\end{array}$ & $\begin{array}{l}2314 \\
2326 \\
2260 \\
\end{array}$ & $\begin{array}{l}1665 \\
1958 \\
2642 \\
\end{array}$ & $\begin{array}{l}1838 \\
2118 \\
3223 \\
\end{array}$ & $\begin{array}{l}1851 \\
1861 \\
1928 \\
\end{array}$ & $\begin{array}{l}2158 \\
2196 \\
1998 \\
\end{array}$ & $\begin{array}{l}2428 \\
2106 \\
2256 \\
\end{array}$ & $\begin{array}{l}2231 \\
2519 \\
2170 \\
\end{array}$ & $\begin{array}{l}2720 \\
2513 \\
2717 \\
\end{array}$ \\
\hline MEAN & & 2380 & 2300 & 2088 & 2393 & 1880 & 2117 & 2263 & 2307 & 2650 \\
\hline
\end{tabular}

results and the mean shown. (The number of function evaluations for convergence of the DFQN method appears to be proportional to $N^{1.8}$.)

It is of interest to observe the detailed behavior of this algorithm for a few cases. In Tables 5 and 6 are shown the results for the Helical Valley and for Rosenbrock's Function. Not only is the convergence clearly superlinear near the solution, but the final estimate "GG" of the Hessian is quite close to that computed by central differences at the solution point.

The output for Powell's function with a quartic minimum is given in Table 7, and shows quite clearly that a method based on quadratic approximation hardly works at all near a higher-order minimum. The convergence is certainly not superlinear (barely linear!), and the final estimate for the Hessian is very far from the differenced estimate (which is very accurate). Oddly enough, the "Hadamard condition number", defined by:

$$
C_{H} \equiv(\operatorname{det} G) /\left(\prod_{i=1}^{N}\left(\sum_{j=1}^{N} G_{i j}^{2}\right)^{1 / 2}\right)
$$

has almost the same value for both estimates. Since $\bar{G}$ is, in reality, singular, the 
conjugacy relations (2.11) become impossible to maintain with sufficient accuracy. Each time such a failure occurs, it is noted, and the total printed in the output, as shown.

TABLE 3

\section{Comparison of DFQN and GMP methods}

\begin{tabular}{|c|c|c|c|c|c|c|c|}
\hline \multirow{2}{*}{\multicolumn{2}{|c|}{ Function }} & \multicolumn{3}{|c|}{ DFQN } & \multicolumn{3}{|c|}{ GMP } \\
\hline & & ITER & EVALS & ACCUR. & ITER & EVALS & ACCUR. \\
\hline Hel & & 23 & 194 & $3.5 \times 10^{-27}$ & 27 & 165 & $2.5 \times 10^{-26}$ \\
\hline Ros & & 25 & 136 & $3.7 \times 10^{-15}$ & 26 & 133 & $2.8 \times 10^{-14}$ \\
\hline Wood & & 25 & 261 & $3.4 \times 10^{-20}$ & 55 & 395 & $4.4 \times 10^{-19}$ \\
\hline Pow & & 43 & 421 & $1.3 \times 10^{-22}$ & 41 & 398 & $1.6 \times 10^{-22}$ \\
\hline Wat & 6 & 24 & 333 & $4.4 \times 10^{-12}$ & 33 & 351 & $1.0 \times 10^{-11}$ \\
\hline Wat & 9 & 69 & 1388 & $1.4 \times 10^{-10}$ & 56 & 939 & $2.8 \times 10^{-10}$ \\
\hline Cheb & 4 & 9 & 105 & $2.8 \times 10^{-18}$ & 8 & 67 & $2.9 \times 10^{-15}$ \\
\hline & 6 & 15 & 232 & $2.3 \times 10^{-17}$ & 13 & 135 & $2.5 \times 10^{-15}$ \\
\hline & 8 & 23 & 487 & $7.9 \times 10^{-14}$ & 20 & 251 & $1.6 \times 10^{-13}$ \\
\hline 20 & 0 & 69 & 3069 & $2.8 \times 10^{-13}$ & 47 & 1189 & $2.5 \times 10^{-1.3}$ \\
\hline Exp & 5 & 61 & 718 & $3.5 \times 10^{-20}$ & 44 & 401 & $4.9 \times 10^{-18}$ \\
\hline Exp & 6 & 42 & 669 & $5.3 \times 10^{-13}(\mathrm{~L})$ & 99 & 978 & $4.1 \times 10^{-18}$ \\
\hline
\end{tabular}

TABLE 4

Comparison of DFQN and Powell's methods on random trigonometric functions

\begin{tabular}{|c|c|c|c|}
\hline & & DFQN & POWELL 1964 \\
\hline Trig & 3 & 72 & 108 \\
\hline Trig & 5 & 191 & 167 \\
\hline Trig. & 10 & 686 & 504 \\
\hline Trig & 20 & 1880 & 2389 \\
\hline \multirow[t]{3}{*}{ Trig } & 50 & 9989 & \\
\hline & " & 15078 & \\
\hline & " & 10943 & \\
\hline
\end{tabular}


TABLE 5

\section{HELICAL VALLEY}

\begin{tabular}{|c|c|c|c|c|c|}
\hline CYCLE & EVALS & $\boldsymbol{F}$ & $x \rightarrow$ & & \\
\hline 0 & 4 & 2.5000503 & $-1.0000 F 00$ & $0.0000 E 00$ & $0.0000 E 00$ \\
\hline 1 & 32 & $2.0216 F 01$ & $-9.2457 E^{-01}$ & $6.2522 E^{-} 01$ & $4.1739 E 00$ \\
\hline 2 & 40 & $1.7257 E 01$ & $8.6814 E^{-} 01$ & $6.1197 E^{-01}$ & $4.0746 E 00$ \\
\hline 3 & 49 & $1.1676 F .01$ & $-4.9033 E^{-} 01$ & $9.9748 E^{-01}$ & $3.2299 E 00$ \\
\hline 4 & 57 & $8.7017 F 00$ & $-2.7873 E^{-01}$ & $9.9702 E^{-01}$ & $2.8815 E 00$ \\
\hline 5 & 65 & $5.5328 F .00$ & $2.6616 E^{-} 01$ & $1.0667 E 00$ & $2.1264 E 00$ \\
\hline 6 & 74 & $4.6213 E 00$ & $4.3775 E^{-} 01$ & $1.0103 E 00$ & $1.8770 E 00$ \\
\hline 7 & 83 & 1.8108500 & $8.4808 E^{-} 01$ & $6.6381 E^{-} 01$ & $\begin{array}{l}1.0132 E 00 \\
8.5569 E\end{array}$ \\
\hline $\begin{array}{l}8 \\
9\end{array}$ & $\begin{array}{r}92 \\
101\end{array}$ & $\begin{array}{l}1.2519 E 00 \\
8.1327 E=01\end{array}$ & $\begin{array}{l}7.9490 E^{-} 01 \\
8.9252 E^{-} 01\end{array}$ & $\begin{array}{l}4.7963 E^{-01} \\
2.9189 E^{-01}\end{array}$ & $\begin{array}{l}8.5569 E^{-} 01 \\
4.5458 E^{-} 01\end{array}$ \\
\hline 10 & 107 & $3.7603 F^{-} 01$ & $9.1868 E^{-} 01$ & $3.6711 E^{-} 01$ & $5.9447 E^{-} 01$ \\
\hline 11 & 113 & $2.4722 E^{-} 01$ & $9.5125 E^{-} 01$ & $2.8770 E^{-} 01$ & $4.4645 E=01$ \\
\hline 12 & 120 & $6.6381 E^{-} 02$ & $9.7831 E^{-} 01$ & $1.4257 E^{-01}$ & $2.2493 E^{-} 0$ \\
\hline 13 & 129 & $3.0043 F^{-} 03$ & $1.0035 F 00$ & $5.8743 E^{-03}$ & $1.3294 E^{-} 02$ \\
\hline 14 & 136 & $1.19 .76 F^{-} 03$ & $1.0023 E 00$ & $1.5532 E^{-02}$ & $2.4799 E^{-} 02$ \\
\hline 15 & 143 & 3857 & $1.0010 F 00$ & $1.1213 E^{-} 02$ & $9 E^{-02}$ \\
\hline 16 & 152 & $1.2269 F^{-04}$ & $1.0003 E 00$ & $4.5152 E^{-03}$ & $6.3285 E^{-} 03$ \\
\hline 17 & 158 & $8.5734 F^{-} 06$ & $1.0000 E 00$ & ${ }^{-} 1.6533 E^{-} 03$ & $-2.7338 E^{-0}$ \\
\hline 18 & 164 & $2.8928 F^{-} 07$ & $1.0000 E 00$ & $-3.3210 E^{-} 04$ & $-5.2372 F^{-} 0$ \\
\hline 19 & 170 & $6.0667 E^{-10}$ & $1.0000 F 00$ & $6.1106 E^{-06}$ & $8.3140 E^{-06}$ \\
\hline 20 & 176 & $3.53947^{-12}$ & $1.0000 E 00$ & $3.1216 E^{-07}$ & $6.5073 E^{-07}$ \\
\hline 21 & 182 & $2.2862 F^{-15}$ & $1.0000 F 00$ & $2.1773 F^{-} 08$ & $-3.7078 E^{-08}$ \\
\hline 22 & 188 & $9.0942 F^{-} 21$ & $1.0000 F 00$ & $2.6327 E^{-11}$ & $3.6231 E^{-11}$ \\
\hline & ONVER & & & & \\
\hline 23 & 194 & $3.4648 F^{-27}$ & 1.0000500 & $-2.4880 E^{-14}$ & $-3.5224 E^{-14}$ \\
\hline
\end{tabular}

GC

$\begin{array}{lcc}200.03 & -0.025331 & 0.014072 \\ -0.025331 & 506.61 & -318.31 \\ 0.014072 & -318.31 & 201.99\end{array}$

GGIT

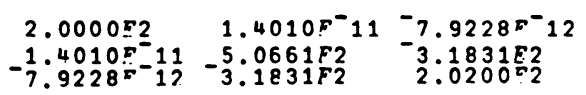

9. Discussion. Although the performance of the DFQN algorithm is creditable enough in most cases, it is clearly inferior to the GMP method for Chebyquad, EXP5 and EXP6.

The possibility of improving this type of algorithm by generalizing it has been outlined by Powell [12]. He terms these methods " $B$-conjugate" methods. 9 The relations (2.11) are retained, but the $\mathrm{QN}$ conditions, instead of being restricted to (3.10b) and (3.14), are generalized by Powell to:

$$
\sum_{i j} C_{i j \sigma} G_{i j}^{*}=r_{\sigma}, \quad \sigma=1, \ldots, m,
$$

where the coefficients $\left\{C_{i j \sigma}\right\}$ and the quantities $\left\{r_{\sigma}\right\}$ are known in terms of values of $x$ and of $f .\left\{G_{i j}^{*}\right\}$ is, of course, required to be symmetric. With these more general QN conditions, for example, it might not be necessary to achieve the conditions (2.6) in the line search, thus rendering it possible to reduce the number of evaluations of $f$. 
TABLE 6

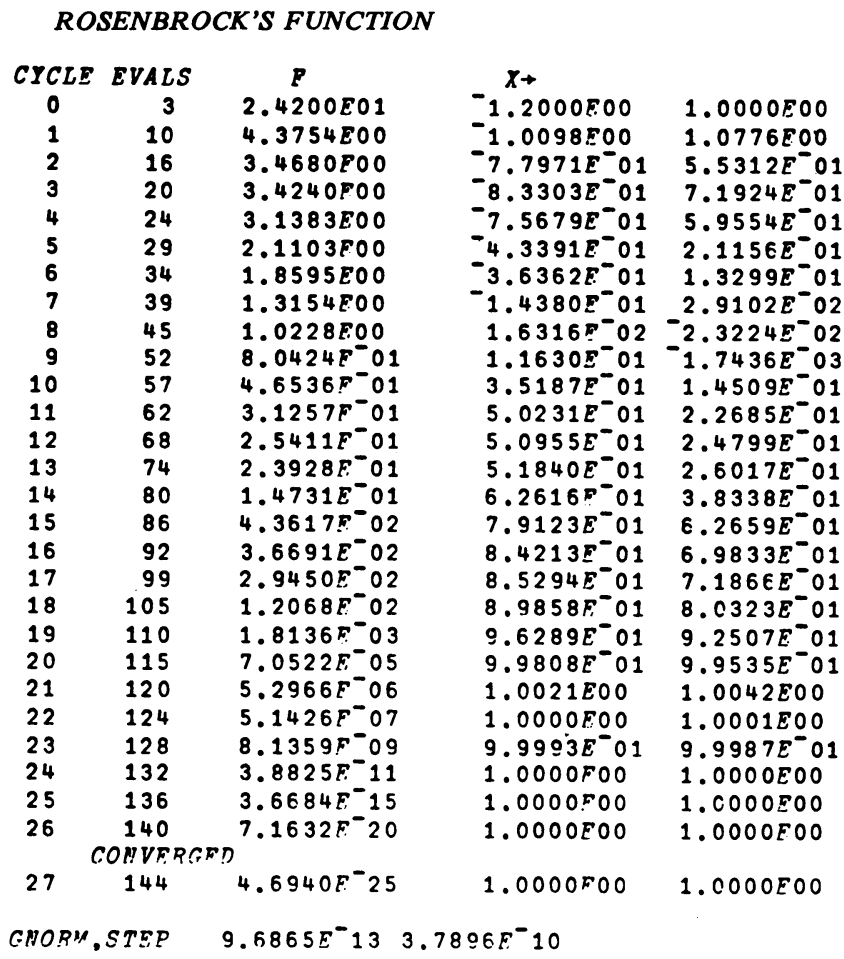

GG

$-801.99-399.99$

$-399.99200$

GTDIF

$-802-400$

$-400 \quad 200$

Powell reported mixed success with an algorithm he devised based on these ideas. His difficulties seemed to be a result of the lack of insurance, in conditions (9.1), that $G^{*}$ would be positive-definite. Moreover, Powell made no provision for estimating $g_{0}^{*}$. If $(9.1)$ is generalized further to:

$$
\sum_{i j} C_{i j \sigma} G_{i j}^{*}+\sum_{i} d_{i \sigma} g_{0 i}^{*}=q_{\sigma}
$$

thus introducing more variables $\left\{g_{0 i}^{*}\right\}$, it would then be possible to constrain $G^{*}$ so as to maintain positive-definiteness, while at the same time having the QN conditions (9.2) strictly satisfied. This might be done along the lines of Section 6 (also suitably generalized); i.e., some norm of $\gamma$ would be minimized, subject to a set of inequality constraints on $G^{*}$. The exact $\mathrm{QN}$ conditions would then be used to complete the solution for the updates. 
TABLE 7

POHELL'S FUNCTION

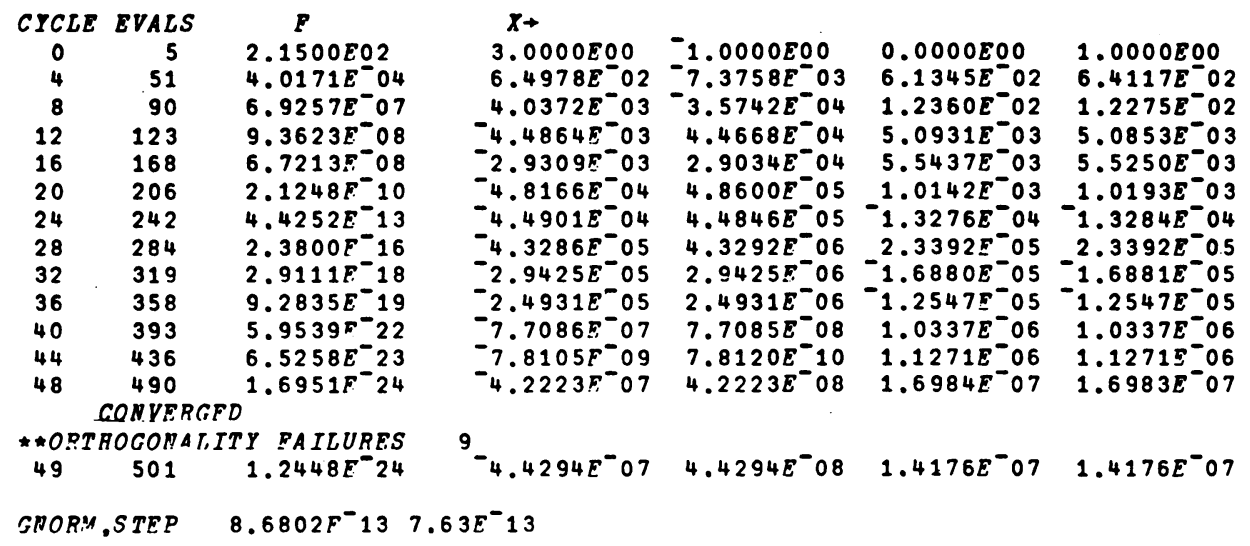

GG

$\begin{array}{rrcc}0.82669 & 8.267 & 1.8316 & -1.8316 \\ 8.267 & 82.671 & 18.317 & -18.317 \\ 1.8316 & 18.317 & 16.94 & -16.94 \\ -1.8316 & -18.317 & -16.94 & 16.94\end{array}$

GCDIF

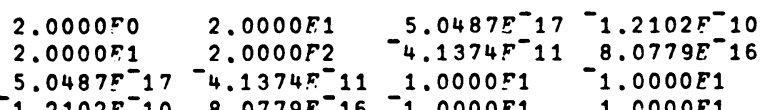

10. Acknowledgments. I am indebted to M. J. D. Powell, S. Schechter, and G. Golub for provocative criticisms and suggestions (some of which have already been mentioned).

Appendix-Line Search. We shall sketch the line search here, touching on the principal precaution for avoiding catastrophes due to rounding error. (There are various other safeguards in the program, but these have little theoretical interest.)

The first phase of the search we term the "trap" phase. Starting with a normalized direction vector $s$, we are evaluating $F(\alpha)$ defined as $f(\tau+\alpha s)$ as described in Section 2. Our first value $(\alpha=0)$, we shall denote by $\alpha_{2}$, and the corresponding value of $F(0)$ by $F_{2}$. We then increment $\alpha$ to the value $\alpha_{3}$, and evaluate $F_{3}$. (If $s$ is the first step direction-viz., the Newton direction, then $\alpha_{3}$ is the value given by the Newton formula; however, in no case is $\alpha_{3}$ permitted to exceed unity. For the other directions in the cycle, $\alpha_{3}$ is estimated on the basis of the progress made in the first step-again, $\alpha_{3}$ cannot exceed unity.)

If $F_{3}<F_{2}$, the step $\alpha_{3}$ is doubled, $\alpha_{2}$ becomes $\alpha_{1}, \alpha_{3}$ becomes $\alpha_{2}$, and a new $\alpha_{3}$ is defined as $\alpha_{2}+2\left(\alpha_{2}-\alpha_{1}\right)$. The function values are also relabeled. $F_{3}$ is next evaluated, and compared with $F_{2}$. If $F_{3}<F_{2}$ another progressive step is made, etc. For some $\alpha_{3}, F_{3}$ will be $\geqslant F_{2}$. In this case, we have "trapped" a smallest value of $F$. 
Now, it can happen that, although $G$ is positive-definite, even the "Newton direction" may not be a descending one, because we have only an estimate of the gradient, and not its true value. Hence, it can always happen that the initial $F_{3}$ is $\geqslant F_{2}$. In this case, we reverse the signs of $s$ and $\alpha_{3}$, denote $\alpha_{3}$ and $F_{3}$ by $\alpha_{1}$ and $F_{1}$, respectively, and make a new step $\alpha_{3}$ in the opposite direction. The new $F_{3}$ may be $<F_{2}$, in which case, we proceed as in the preceding paragraph. Otherwise, we again have "trapped" the smallest value $F_{2}$.

Under certain circumstances, this would end the line search. However, there may be certain unsatisfactory conditions that necessitate a more refined "squeeze" of the middle point $\left(\alpha_{2}, F_{2}\right) .^{10}$ These are:

(a) It is the first step of the cycle and $\alpha_{2}=0$. (This might result in a null step for the entire cycle, thus unnecessarily terminating the algorithm.)

(b) The estimate of $c$ gained from the $\alpha$ 's and $F$ 's via the method of Section 3 exceeds 10. (Because of the normalization of the $\left\{s_{i}\right\}$, the value of $c$ becomes very nearly unity near the solution. For this reason, large estimates of $c$ are suspect, since a very bad value for $c$ can render it very difficult or impossible to recover good estimates $g$ and $G$ during later cycles.)

The "squeeze" itself is based on first fitting a quadratic to the three points $P_{1}, P_{2}$, and $P_{3}$. The minimum of this quadratic will occur at $\alpha_{4}$, with $\alpha_{1}<\alpha_{4}<$ $\alpha_{3}$. When $F_{4}$ is now evaluated it may be $\geqslant F_{2}$. In this case, we perform a "cut", i.e., if, for example, $\alpha_{4}>\alpha_{2}$, we compute ${ }^{11}$

$$
\alpha_{5}=1 / 2\left(\alpha_{1}+\alpha_{2}\right)
$$

and "close" the interval, by discarding $P_{3}$. Then $P_{4}$ becomes $P_{3}$, and we evaluate $F_{5} ; P_{5}$ then becomes the new $P_{4}$. If $F_{4}$ is again $>F_{2}$, we repeat the process. Note that the "cut" is always on the side away from $P_{4}$.

When $F_{4}<F_{2}$, we fit a cubic to the four points $P_{1}, P_{2}, P_{3}, P_{4}$. Let this cubic be centered around $\alpha_{4}$ as follows:

$$
\kappa(\alpha)=c_{0}+c_{1}\left(\alpha-\alpha_{4}\right)+c_{2}\left(\alpha-\alpha_{4}\right)^{2}+c_{3}\left(\alpha-\alpha_{4}\right)^{3}
$$

(with the $c$ 's having known values after the fitting). We can then solve for the minimum of $\kappa(\alpha)$, and we obtain the solution (for $c_{2} \neq 0$ ):

$$
\alpha_{5}=\alpha_{4}-\frac{c_{1}}{c_{2}}\left(\frac{1}{1+\sqrt{1-\rho}}\right)
$$

where

$$
\rho \equiv 3 c_{1} c_{3} / c_{2}^{2}
$$

$\rho$ is a dimensionless ratio, independent of the scaling of $F$ or $\alpha$, and, for a cubic, is bounded above by unity.

10 We shall henceforth denote the pair $\left(\alpha_{i}, F_{i}\right)$ by $P_{i}$.

${ }^{11}$ This device was originally suggested to the author by Dr. Y. Bard. 
The criterion for terminating the "squeeze" is based on the relative change in the estimated value of $F^{\prime \prime}(\alpha)$ from $\alpha_{4}$ to $\alpha_{5}$. In this case, the estimates are based on $\kappa(\alpha)$, and the values of $\kappa^{\prime \prime}$ at $\alpha_{4}$ and $\alpha_{5}$ turn out to be:

$$
\begin{aligned}
& \kappa^{\prime \prime}\left(\alpha_{4}\right)=2 c_{2}, \\
& \kappa^{\prime \prime}\left(\alpha_{5}\right)=2 c_{2} \sqrt{1-\rho},
\end{aligned}
$$

so that:

$$
\kappa_{5}^{\prime \prime} / \kappa_{4}^{\prime \prime}=\sqrt{1-\rho} .
$$

It can be shown that, when the values $\left\{\alpha_{1}, \alpha_{3}\right\}$ do not bracket the maximum of $\kappa(\alpha)$, then $\kappa_{5}^{\prime \prime}$ will be larger than $\kappa_{4}^{\prime \prime}$. Hence, we can expect that the "normal" state of affairs would be that the ratio in (A6) would be greater than unity, which means that $\rho$ would be negative. Numerical tests have indicated that it is in fact reasonable to allow $\kappa "$ to increase by $20 \%$ but to restrict any decrease to $1 \%$. This gives an allowable range for $\rho$ as follows:

$$
-.44<\rho<.02
$$

and when $\rho$ is found to fall within this range, the squeeze is terminated.

The principal danger from rounding error occurs when the differences $\left(F_{1}-F_{2}\right)$ and $\left(F_{3}-F_{2}\right)$ are too small relative to $\left|F_{2}\right|$. Then, too many significant figures are lost, and the values of $b$ and $c$ become too inaccurate. This has the effect of spoiling the updates for $g$ and $G$. Therefore, since the machine accuracy in this study is about 16 significant figures, the line search is terminated and no update is made when,

$$
\min \left(F_{1}-F_{2}, F_{3}-F_{2}\right)<10^{-12} \times F_{2}
$$

so that we can expect at least a few correct figures in our update.

IBM Corporation

Palo Alto Scientific Center

1530 Page Mill Road

Palo Alto, California 94304

1. J. GREENSTADT, Math. Comp., vol. 26, 1972, p. 145.

2. M. MARCUS \& H. MINC, A Survey of Matrix Theory and Matrix Inequalities, Allyn and Bacon, Boston, Mass., 1964.

3. R. FLETCHER \& M. J. D. POWELl, Comput. J., vol. 6, 1963, p. 163.

4. H. H. ROSENBROCK, Comput. J., vol. 3, 1960, p. 175.

5. A. R. COLVILlE, A Comparative Study of Non-Linear Programming Codes, IBM NY Scientific Center Report \#320-2949, 1968.

6. M. J. D. POWELL, Comput. J., vol. 5, 1962, p. 147.

7. R. P. BRENT, Algorithms for Finding Zeros and Extrema of Functions Without Calculating Derivatives, Stanford Univ. Comput. Sci. Report STAN-CS-71-198, 1971.

8. R. FLETCHER, Comput. J., vol. 8, 1965, p.33.

9. M. C. BIGGS, J. Inst. Math. Appl., vol. 8, 1972, p. 315.

10. P. E. GILL, W. MURRAY \& R. A. PITFIELD, The Implementation of Two Revised Quasi-Newton Algorithms for Unconstrained Optimization, Nat. Phys. Lab. Report NAC 11 , 1972.

11. M.J.D. POWELL, Comput. J., vol. 7, 1964, p. 155.

12. M. J. D. POWELL, ACM Trans. Math. Software, vol. 1, 1975, p. 97. 